\title{
Poster Abstract: Design Considerations for Low Power Internet Protocols
}

\author{
Hudson Ayers \\ Stanford University \\ Palo Alto, California \\ hayers@stanford.edu \\ Conor McAvity \\ Stanford University \\ Palo Alto, California \\ cmcavity@stanford.edu
}

\author{
Paul Thomas Crews \\ Stanford University \\ Palo Alto, California \\ ptcrews@stanford.edu \\ Amit Levy \\ Stanford University \\ Palo Alto, California \\ levya@cs.stanford.edu
}

\author{
Hubert Hua Kian Teo \\ Stanford University \\ Palo Alto, California \\ hteo@stanford.edu \\ Philip Levis \\ Stanford University \\ Palo Alto, California \\ pal@stanford.edu
}

\begin{abstract}
Examining implementations of the 6LoWPAN Internet Standard in major embedded operating systems, we observe that they do not fully interoperate. We find this is due to some inherent design flaws in 6LoWPAN. We propose and demonstrate four principles that can be used to structure protocols for low power devices that encourage interoperability between diverse implementations.
\end{abstract}

\section{CCS CONCEPTS}

- Networks $\rightarrow$ Network protocols; $\bullet$ Computer systems organization $\rightarrow$ Sensor networks;

\section{KEYWORDS}

6LoWPAN, Internet of Things, Interoperability

ACM Reference Format:

Hudson Ayers, Paul Thomas Crews, Hubert Hua Kian Teo, Conor McAvity, Amit Levy, and Philip Levis. 2018. Poster Abstract: Design Considerations for Low Power Internet Protocols. In SenSys '18: Conference on Embedded Networked Sensor Systems, November 4-7, 2018, Shenzhen, China. ACM, New York, NY, USA, 2 pages. https://doi.org/10.1145/3274783.3275161

\section{INTRODUCTION}

Interoperability is critical for the Internet of Things. To address this, the IETF published the 6LoWPAN Internet Standard, which specifies how to format IPv6 packets over low-power wireless links such as $802.15 .4[5,6]$, including a fragmentation format and aggressive compression. These standards have been implemented by a number of popular embedded operating systems, including Contiki [4], RiotOS [3], OpenThread [7], mbedOS [2], and TinyOS [1].

One key feature of 6LoWPAN is its many mechanisms for compressing IPv6 packets. For example, a sender may elide 14 bytes of an address if it carries the link-local prefix. Senders are not required to optimally compress packets, but receivers need to decompress

Permission to make digital or hard copies of all or part of this work for personal or classroom use is granted without fee provided that copies are not made or distributed for profit or commercial advantage and that copies bear this notice and the full citation on the first page. Copyrights for components of this work owned by others than ACM must be honored. Abstracting with credit is permitted. To copy otherwise, or republish, to post on servers or to redistribute to lists, requires prior specific permission and/or a

fee. Request permissions from permissions@acm.org.

SenSys '18, November 4-7, 2018, Shenzhen, China

(C) 2018 Association for Computing Machinery.

ACM ISBN 978-1-4503-5952-8/18/11 ..\$15.00

https://doi.org/10.1145/3274783.3275161
Table 1: 6LoWPAN Interoperability Matrix

\begin{tabular}{lccccc}
\hline Feature & \multicolumn{5}{c}{ Stack } \\
\hline Uncompressed IPv6 & $\checkmark$ & & $\checkmark$ & $\checkmark$ & $\checkmark$ \\
Fragmentation & $\checkmark$ & $\checkmark$ & $\checkmark$ & $\checkmark$ & $\checkmark$ \\
1280 byte packets & $\checkmark$ & $\checkmark$ & $\checkmark$ & $\checkmark$ & $\checkmark$ \\
Dispatch_IPHC hdr & $\checkmark$ & $\checkmark$ & $\checkmark$ & $\checkmark$ & $\checkmark$ \\
Stateless Addr Comp. & $\checkmark$ & $\checkmark$ & $\checkmark$ & $\checkmark$ & $\checkmark$ \\
Stateless + multicast & $\checkmark$ & $\checkmark$ & $\checkmark$ & $\checkmark$ & $\checkmark$ \\
802.15.4 16 bit addr & & $\checkmark$ & $\checkmark$ & $\checkmark$ & $\checkmark$ \\
Address Autoconfig & $\checkmark$ & $\checkmark$ & $\checkmark$ & $\checkmark$ & $\checkmark$ \\
Stateful Addr Comp. & $\checkmark$ & $\checkmark$ & $\checkmark$ & $\checkmark$ & $\checkmark$ \\
Stateful + multicast & & $\checkmark$ & $\checkmark$ & $\checkmark$ & \\
TC/Flow Label comp. & $\checkmark$ & $\checkmark$ & $\checkmark$ & $\checkmark$ & $\checkmark$ \\
Tunneled IPv6 Comp. & & $\checkmark$ & & $\checkmark$ & $\checkmark$ \\
IPv6 NH Comp: UDP & $\checkmark$ & $\checkmark$ & $\checkmark$ & $\checkmark$ & $\checkmark$ \\
UDP port comp. & $\checkmark$ & $\checkmark$ & $\checkmark$ & $\checkmark$ & $\checkmark$ \\
UDP cksum elision & & & & & $\checkmark$ \\
Headers past first frag & & & $\checkmark$ & $\checkmark$ & \\
Extension Hdr comp. & & $\sim$ & & $\checkmark$ & $\checkmark$ \\
Mesh Header & & $\checkmark$ & & $\checkmark$ & $\sim$ \\
Broadcast Header & & & & & $\checkmark$ \\
Regular IPv6 ND & $\checkmark$ & & $\checkmark$ & $\checkmark$ & $\sim$ \\
RFC 6775 6Lo ND & & & $\checkmark$ & $\checkmark$ & \\
RFC 7400 GHC & & & & & \\
\hline ～Partial Support & & & & &
\end{tabular}

$\sim=$ Partial Support

any received packet regardless of which compression mechanisms are used (or not).

\section{INTEROPERABILITY STUDY}

None of the five 6LoWPAN implementations we examined is able to decode every possible 6LoWPAN packet. Moreover, implementations differ in which portions of the specification they choose to support. Table 1 summarizes our findings.

To further verify the findings of Table 1 , we verified that for each of the 10 possible pairings of 6LoWPAN stacks, a single example packet can be generated by one which the other would not receive. We also tested a subset of these pairings by flashing the operating systems onto embedded hardware and sending various 6LoWPAN 
Table 2: 6LoWPAN Stack Code Size

\begin{tabular}{lrrrrr}
\hline Stack & \multicolumn{5}{c}{ Code Size Measurements (Bytes) } \\
\hline \multicolumn{7}{r}{ Full IP } & 6Lo & Comp. & Frag. & Mesh/B-Cast \\
\hline Contiki & 37538 & 11262 & 5952 & 3319 & N/A \\
OT & 42262 & 26375 & $>15000$ & 1310 & 4500 \\
Riot & 30942 & 7500 & $>4712$ & 1514 & N/A \\
Arm & 46030 & 22061 & 17900 & 3104 & 1331 \\
TinyOS & 37312 & 16174 & -- & -- & 600 \\
\hline * TinyOS inlines compression, doesn't implement full mesh
\end{tabular}

messages - when doing so, we observed that packet drops matched our expectations.

Beyond this mismatched feature implementation, we also discovered significant implementation-specific variations. Two prime examples of this include varied bounds on the maximum header decompression possible for a received 6LoWPAN packet, and varied bounds on the extent to which arbitrary next header compression is allowed. In both cases, these variations led to additional interoperability failures, and were due to independent decisions made by designers to conserve code space or RAM.

\section{CONTRIBUTING FACTORS}

The 6LoWPAN specification was created with a clear goal-to bring IPv6 to embedded devices while keeping radio utilization competitive with prior low power wireless protocols. The irony is that if too much of an emphasis is put on compression mechanisms to save energy, the resulting increase in code requires more expensive, power-hungry microcontrollers. In practice, many 6LoWPAN implementations do not implement the entire specification and, therefore, are not interoperable. This is not a result of poor software design, but rather intentional choices to implement different subsets of the specification that favor limited RAM and code size and minimizing engineering effort. Illustrating this, several implementations include compile-time options to remove portions of the 6LoWPAN stack during compilation. For example, Contiki defines the SICSLOWPAN_CONF_COMPRESSION compilation flag, which can be set to force all Contiki packets to be processed as uncompressed IPv6. Riot presents even more extensive compilation options for 6LoWPAN.

Table 2 shows the code size overhead of each of the five implementations broken into independent overheads for compression, fragmentation, mesh and broadcast headers, as well as totals for 6LoWPAN and the entire IPv6 stack. Key takeaways from Table 2:

- Fragmentation, the only portion of 6LoWPAN that's strictly necessary, consumes much less ROM than compression

- Implementations with more complete adherence to the compression specification consume more code for compression

These implementation concerns reveal two primary factors which contributed to 6LoWPAN's interoperability problems:

- Trouble balancing code size vs. compression

- Blind belief that embedded developers would implement entirety of a complex specification

\section{FOUR DESIGN PRINCIPLES}

We propose four protocol design principles which, if followed, lead to low-power protocols that are more likely to have interoperable implementations:

\section{Principle 1: Capability Spectrum}

Rather than require that every device pay the energy costs of reduced complexity, a protocol should support a spectrum of device capabilities which defines a clear ordering to reduce code and RAM size. This capability spectrum should be a linear scale. For a device to support capability level $N$, it must also support all lower levels. This minimizes the cost/complexity of communicating these capabilities. Application to 6LoWPAN: Replace the large collection of "features" in Table 1 with a small number of levels of functionality. These levels prioritize packet size savings vs. implementation complexity. Principle 2: Capability Discovery

There should be a mechanism by which two devices can discover what capability level to use when they communicate. A pair of devices defaults to the lower of their supported capability levels. Application to 6LoWPAN: 6LoWPAN could implement capability negotiation using two mechanisms: neighbor discovery (ND) and ICMP. The ICMP option is particularly important, as it would prevent all of the silent packet drops that currently occur.

\section{Principle 3: Provide Reasonable Bounds}

To bound RAM use, specifications should specify reasonable bounds on recursive or variable features. These bounds ensure that capability negotiation is sufficient to interoperate.

Application to 6LoWPAN: Maximum header decompression should be bounded to 50 bytes. Compressed IPv6 extention options should be ordered, and tunneled IPv6 should never be compressed.

\section{Principle 4: Don't Break Layering}

By breaking layering, cross-layer energy optimizations require that developers own and customize the entire software stack, which is infeasible for modern embedded systems.

Application to 6LoWPAN: Remove UDP checksum compression.

\section{CONCLUSION}

Interoperability is critical to bringing Internet connectivity to the next 100 million devices. To enable this, low power protocols must be intentionally designed to support interoperability between varied devices. The principles in this poster provide a framework for designing protocols with interoperability baked into the design.

\section{ACKNOWLEDGMENTS}

This work was supported in part by Intel/NSF CPS Security grant \#1505728, with additional support from a Stanford Graduate Fellowship, VMware, Google, and the Secure Internet of Things Project.

\section{REFERENCES}

[1] TinyOS Alliance. 2018. TinyOS. https://github.com/tinyos/tinyos-main. (2018).

[2] ARM. 2018. ARM Mbed OS. https://github.com/ARMmbed/mbed-os. (2018).

[3] FU Berlin. 2018. Riot OS. https://github.com/RIOT-OS/RIOT. (2018).

[4] Adam Dunkels. 2018. Contiki OS. https://github.com/contiki-os/contiki. (2018).

[5] J. Hui and P. Thubert. 2011. Compression Format for IPv6 Datagrams over IEEE 802.15.4-Based Networks. RFC 6282. RFC Editor. http://www.rfc-editor.org/rfc/ rfc6282.txt http://www.rfc-editor.org/rfc/rfc6282.txt.

[6] G. Montenegro, N. Kushalnagar, J. Hui, and D. Culler. 2007. Transmission of IPv6 Packets over IEEE 802.15.4 Networks. RFC 4944. RFC Editor. http://www.rfc-editor. org/rfc/rfc4944.txt http://www.rfc-editor.org/rfc/rfc4944.txt.

[7] Nest. 2018. OpenThread. https://github.com/openthread/openthread. (2018). 